INVESTIGACIÓN

\title{
DIAGNÓSTICO SITUACIONAL DEL HATO LECHERO UFPSO CON RESPECTO A LOS LINEAMIENTOS DE PRODUCCIÓN MÁS LIMPIA
}

\section{SITUATIONAL DIAGNOSIS UFPSO DAIRY HERD WITH RESPECT TO THE GUIDELINES FOR CLEANER PRODUCTION}

\author{
Ing. Juan David Herrera Galviz a , MSc. Juan Carlos Hernández Criado ${ }^{\text {, }}$, MSc. José Arnoldo \\ Granadillo Cuello ${ }^{\mathrm{c}}$
}
âniversidad Francisco de Paula Santander Ocaña, Grupo de Investigación GI@DS. Vía Acolsure Sede El Algodonal, Ocaña - Norte de Santander, Colombia. Email: jdherrerag@ufpso.edu.co
bUniversidad Francisco de Paula Santander Ocaña, Grupo de Investigación GI@DS. Vía Acolsure Sede El Algodonal, Ocaña - Norte de Santander, Colombia. Email: jchernandezc@ufpso.edu.co
'Universidad Francisco de Paula Santander Ocaña, Grupo de Investigación GI@DS. Vía Acolsure Sede El Algodonal, Ocaña - Norte de Santander, Colombia. Email: jagranadilloc@ufpso.edu.co

Fecha de recepción: 02-10-2016

Fecha de aprobación: 03-12-2016

\begin{abstract}
Resumen: El objetivo principal del trabajo es la realización de un diagnóstico situacional ambiental del proyecto Hato Lechero de la Universidad Francisco de Paula Santander Ocaña, a partir del empleo de algunas metodologías para la recolección de datos, con el fin de analizar la información recopilada, y poder formular algunas acciones para la implementación de producción más limpia. Básicamente se empleó una entrevista como primer instrumento, complementándose con una lista de chequeo, para proceder a realizar una matriz de identificación y valoración de impactos ambientales, y posteriormente el flujo de procesos del proyecto. Lo anterior permitió realizar un análisis DOFA, y proponer posibles soluciones a las problemáticas halladas. Como resultado, se pudo determinar que el proyecto presenta varias falencias en cuanto a la gestión ambiental dentro de sus actividades, y principalmente resalta la falta de registro y control de sus materias primas y de sus residuos, por lo que no se realiza una gestión adecuada de los mismos, lo que repercute en la degradación del suelo por residuos sólidos y líquidos y la degradación de la calidad del aire por emisión de gases de efecto invernadero. Se concluye que el proyecto tiene varias falencias, pero también tiene unas
\end{abstract}


fortalezas importantes que debe aprovechar para adentrarse en la producción más limpia, mejorando su gestión ambiental.

Palabras clave: Gestión Ambiental, Hato Lechero, Materias Primas, Producción Más Limpia, Residuos.

\begin{abstract}
The main objective of the work is to carry out an environmental situational diagnosis of the dairy herd project of the Universidad Francisco de Paula Santander Ocaña, based on the use of some methodologies for data collection, in order to analyze the information collected, Formulate some actions for the implementation of cleaner production. Basically, an interview was used as the first instrument, complemented by a checklist, to proceed with a matrix of identification and assessment of environmental impacts, and subsequently the flow of project processes. This allowed for a DOFA analysis, and propose possible solutions to the problems encountered. As a result, it could be determined that the project has several shortcomings in terms of environmental management within its activities, and mainly highlights the lack of registration and control of its raw materials and waste, so that proper management Of them, which affects the degradation of the soil by solid and liquid waste and the degradation of air quality by emission of greenhouse gases. It concludes that the project has several shortcomings, but also has important strengths that it must take advantage of to enter into the cleaner production, improving its environmental management.
\end{abstract}

Keywords: Environmental Management, Dairy Cattle, Raw Materials, Cleaner Production, Waste.

\section{INTRODUCCIÓN}

La Gestión Ambiental en las empresas se evidencia a través de su Sistema de Gestión Ambiental, el cual le ayuda a ser más efectiva para poder lograr sus metas ambientales a partir de una política y objetivos que han sido establecidos desde el direccionamiento general de la misma, lo que se constituye en un gran esfuerzo que de manera organizada, amplia y permanente, permite el mejoramiento de su desempeño ambiental, ofreciendo ventajas como: adaptación a la realidad de la empresa y su gestión general, respaldo de una organización internacionalmente reconocida y aceptada, consistencia en la política de Desarrollo Sostenible, con las regulaciones y exigencias de los consumidores, mejor acceso a seguros, permisos y otras autorizaciones, reducción de los riesgos de operación y accidentes laborales, mayor utilización de los recursos para reducir los costos operativos y mejoramiento de la imagen ante los consumidores y la comunidad en general (Guédez-Mozur et al, 2003).

Según Penella (2012), la producción más limpia mejora la eficiencia y reduce los riesgos para la salud humana y para el ambiente, mediante reducción en cuanto al consumo de materias prima, agua y energía, así como también la eliminación de insumos peligros y la disminución de la cantidad y toxicidad de emisiones y residuos en la fuente, y es así como se considera que la producción más limpia incorpora la variable ambiental en el concepto de calidad de una empresa. 
Varón (2013), recalca que la gestión ambiental en Colombia nació a partir de la necesidad de cambiar nuestro sistema económico basado en un consumismo desenfrenado y como respuesta a los retos que se asumieron a nivel internacional desde las cumbres de la tierra de 1972 y 1992 , y es a partir de este momento donde surge la Producción Más Limpia como la Principal estrategia dentro de la Gestión Ambiental de nuestro país.

Actualmente existe una gran demanda de patrones sostenibles de producción, a través de todas las evidencias ambientales presentes, las políticas y acuerdos, los mercados y la sociedad. Dichos Patrones propenden por reducir la contaminación y favorecer la integridad ambiental, mediante los principios de competitividad empresarial, productividad y eficiencia, generándose dinámicas sectoriales que permiten la creación y afianzamiento de la cultura de producción y consumo racional en los ganaderos del país (SENA, 2010).

Las fincas que se dedican a la producción de leche, en algunas ocasiones se ven afectadas en cuanto a la poca demanda del producto, debido a sus problemas de contaminación del ambiente, ya que se considera a este sector como altamente contaminante, evidenciándose en muchos casos en los que se le ha dado un manejo inadecuado a las excretas de los animales y a otros residuos, aunque claro está, que una finca bien administrada o muy organizada, puede implementar producción más limpia, logrando conseguir una responsabilidad ambiental (Camacho-Fidalgo, 2011).

La FAO (2012), en su guía de buenas prácticas en explotaciones lecheras, recomienda que para realizar una real gestión medioambiental se debe partir de 3 tipos de buenas prácticas, las cuales son: implementar un sistema de gestión agrícola sostenible, disponer de un sistema adecuado de residuos, cerciorarse de los posibles efectos negativos que pueda tener las actividades de la explotación lechera en el medio ambiente local, y esto se realiza a través de unos objetivos basados en el cumplimiento de la legislación ambiental y las expectativas de la comunidad, en la reducción del potencial del impacto negativo de la explotación y en el fortalecimiento de una imagen positiva de la misma.

Dentro de los beneficios que contempla la introducción de prácticas de producción más limpia en los hatos lecheros, se destacan la disminución de los costos de tratamiento y disposición de residuos, valorización de los residuos para su venta como insumos, disminución en costos de limpieza y mantenimiento, reducción en costos producto de problemas de salud ocupacional, ahorro de agua y energía y por consiguiente en pago de tarifas, mejor oportunidad de mejoramiento empresarial y logro de objetivos de calidad del producto, reconversión tecnológica y mejoramiento de las relaciones con la comunidad campesina aledaña y la imagen pública en general (Garzón-Benavides y López-Morán, 2008).

En Colombia existe una gran cantidad de legislación que aplica al Sector de la Ganadería Bovina y específicamente al tema de la producción de leche. El decreto 1541 de 1978, es el que reglamenta el tema de concesiones de aguas, para todo tipo de usos (Presidencia de la República de Colombia, 1978). La ley 373 de 1997, que reglamenta el programa de uso y ahorro eficiente del agua (Congreso de Colombia, 1997). La resolución 0322 de 2004 que reglamente todo lo concerniente los mínimos requisitos sanitarios que se deben considerar en las fincas dedicadas a diferentes explotaciones pecuarias (ICA, 2004). La ley 84 de 1989 
que reglamenta mediante un estatuto nacional, todo lo relacionado con protección de los animales y/o bienestar animal (Congreso de la República, 1989).

La legislación mayormente relacionada con la parte ambiental y aplicable al sector, se encuentran: La ley 99 de 1993 donde se establecen todas las disposiciones de uso, aprovechamiento y protección de los recursos naturales (Congreso de Colombia, 1993).El Decreto 2811 de 1974, el cual se considera como el código de los recursos naturales (Presidente de la República, 1974). La ley 430 de 1998, donde se reglamenta todo lo correspondiente a residuos peligrosos (Congreso de Colombia, 1998). Y la ley 9 de 1979, considerado el código nacional sanitario (Congreso de Colombia, 1979).

Con el fin de verificar y realizar diagnósticos situacionales ambientales para monitorear la gestión ambiental de las empresas y prácticas de producción más limpia, se utilizan las listas de chequeo y según lo dispuesto por Bichachi (2003), estas herramientas son muy utilizadas especialmente para verificar el cumplimiento de la ley en distintas disciplinas. Por otra parte es muy frecuente el uso de la metodología de los ecomapas, ya que para Medina y Zea (2005), este permite la identificación de las principales operaciones y situaciones de carácter crítico que se observan en los procedimientos y actividades de un sector en específico. Otra metodología, muy utilizada en los diagnósticos son las matrices de identificación y valoración de impactos ambientales, en donde Arboleda (2008) recalca que son metodologías de doble entrada, utilizadas para relacionar aspectos $\mathrm{y}$ factores que pueden provocar impactos, y que como producto de esta interacción se obtienen la determinación de los impactos ambientales a partir de la comprensión de las relaciones causa-efecto. A su vez este mismo autor recalca que en procesos de caracterización y diagnóstico se utilizan los flujos de procesos para determinar las actividades que interactúan con el ambiente, a partir de la identificación de los puntos donde estas se llevan a cabo.

Uno de los trabajos de diagnóstico y formulación de estrategias de producción más limpia, fue el realizado por Espitia (2010), el cual desarrolló una propuesta de gestión ambiental basada en las herramientas de PML, para la empresa Siprocas en uno de sus hatos lecheros ubicados en el municipio de Corozal (Casanare), en donde empleó las metodologías de identificación y valoración de impactos ambientales mediante matrices, determinación de puntos críticos de contaminación mediante Ecomapa, y determinación de flujos de procesos y balance de materia y energía, información que utilizó para formular las respectivas estrategias mediante un plan de acción, en las cuales hizo énfasis en el manejo de potreros, debido a que la empresa presentaba una falencia importante y representativa en este aspecto.

La ganadería bovina es una de las actividades que más impacta negativamente al recurso hídrico, debido a las actividades que desarrolla, como la producción de leche, afectando su cantidad y calidad (Loaiza y Osorio, 2009). La problemática ambiental del sector de la producción de leche se enfoca principalmente al manejo de los residuos líquidos los cuales están constituidos por grasas, aceites, sólidos suspendidos y nitrógeno amoniacal, con una alta presencia de materia orgánica, que fluctúa el pH y la temperatura, además de presentarse altos niveles de fosforo $y$ nitrógeno. Sin embargo también se pueden 
encontrar residuos sólidos producto de la actividad del sector, donde se prioriza todo lo relacionado con productos vencidos, maderas, papeles y plásticos que se usan en el envasado de materias primas y en el producto como tal (Isaza-Vargas, 2012).

La Granja de la Universidad actualmente presenta una problemática ambiental debido a la generación de distintos tipos de residuos que en su mayoría no son tratados de la forma más adecuada, desencadenando contaminación por vertimientos, residuos sólidos y emisiones gases. Dentro de la granja experimental uno de los proyectos de mayor relevancia es el Hato Lechero debido a su dimensión, ya que representa por obvias razones los mayores consumos de materias primas e insumos de toda la granja, generando la mayor cantidad de residuos y por consiguiente la mayor contaminación e impacto al medio ambiente

La importancia de esta investigación radica en la necesidad que existe de implementar estrategias de gestión ambiental en la granja experimental de la Universidad, con el fin de poder ofrecer productos a la comunidad de calidad, y a su vez mejorar la imagen ante la comunidad académica, específicamente ante los estudiantes de zootecnia e Ingeniería Ambiental, así como también ante la comunidad en general, contribuyendo a la viabilización de una Granja autosostenible, ejemplo a seguir para los productores agropecuarios de la región. Para ello es necesario realizar este tipo de trabajos, donde inicialmente se conozcan la situación en la que se encuentran los proyectos de la Granja y específicamente el proyecto Hato Lechero, donde se pueda deducir los principales problemas que se presentan con el fin de generar soluciones pertinentes. Es por ello que este tipo de investigaciones propenden al dimensionamiento de la gestión ambiental, a partir de la determinación de los aspectos ambientales que están produciendo impactos ambientales negativos, y que se deben solucionar, en este caso, a partir de la formulación y el empleo de la producción más limpia como principal herramienta.

El presente trabajo, tiene como objetivo la realización de un diagnóstico situacional preliminar para el Hato Lechero de la Universidad Francisco de Paula Santander Ocaña Sede El Algodonal, infraestructura que hace parte de la Granja Experimental de la Institución y que además constituye gran parte del proyecto Bovino de la misma, con el fin de poder conocer la situación en la que se encuentra el proyecto y las falencias de tipo ambiental que presenta, para poder realizar un análisis que permita establecer las medidas a seguir para formulación e implementación de la Producción Más Limpia en el proyecto.

\section{METODOLOGÍA}

La Granja Experimental UFPSO se ubica a la margen derecha del río Algodonal, dentro del campus universitario, a una altura de $1150 \mathrm{msnm}$, con una temperatura promedio de $23{ }^{\circ} \mathrm{C}$, una humedad relativa del $70 \%$ y una extensión de 135 ha; también cuenta con el Centro de Investigación La Troya, que se encuentra ubicado en el corregimiento de Los Ángeles (Río de Oro - Cesar), dedicada al estudio de ganado de las razas Romosinuano y Costeño con Cuernos. Dentro de los proyectos o explotaciones pecuarias que se encuentran, está el Hato Lechero el cual desarrolla la línea de ejemplares especializados para la producción láctea, conformada por vientres de raza Gyrolando, con producción promedio de 18 lt/vaca diarios. 
Para conocer a profundidad el funcionamiento del hato lechero de la Universidad Francisco de Paula Santander Ocaña, se realizó inicialmente una entrevista al coordinador del mismo. La entrevista básicamente contenía las preguntas que se visualizan en la Tabla 1.

\begin{tabular}{l} 
Preguntas \\
\hline ¿Qué razas de bovinos posee el hato? \\
¿Cuántos animales están a disposición del hato lechero? \\
¿Cuál es la producción diaria de leche por animal? \\
¿Cuánta agua es requerida para el consumo de cada animal? \\
¿Cuál es la cantidad total de alimento que consumen \\
diariamente los animales? \\
¿Qué tipos o especies de forrajes consumen los animales? \\
¿Cuáles son los horarios de ordeño? \\
¿Qué equipos son requeridos para el funcionamiento del \\
hato? \\
¿Cuánto tiempo permanecen encendidas las luces? \\
¿Cuántos potreros tienen a disposición para el pastoreo de \\
los animales? \\
¿Realizan rotación de potreros? ¿Cada cuánto? \\
¿Realizan control de malezas en los potreros? ¿De qué \\
manera y con qué frecuencia? \\
¿Cómo se maneja el aspecto zoosanitario en el hato \\
lechero? \\
¿Tienen conocimiento de los consumos de agua y energía \\
eléctrica del hato lechero? \\
¿Qué sustancias contienen los residuos líquidos generados \\
por el proyecto? \\
¿Dónde se ubican los puntos de vertimientos de residuos \\
líquidos? \\
¿Se realiza algún tratamiento a los residuos líquidos \\
generados? \\
¿Qué tipo de residuos sólidos son generados? \\
¿Dónde se ubican los puntos de generación de residuos \\
sólidos? \\
¿Se realiza algún tratamiento a los residuos sólidos \\
generados? \\
¿Cuál es el manejo que se les da a los residuos peligrosos \\
generados por el proyecto? \\
¿Llevan ustedes un control de la cantidad de residuos \\
sólidos, líquidos, gaseosos y peligrosos generados por el \\
hato lechero? \\
En promedio, ¿Cuánta agua es utilizada para las labores de \\
lavado y desinfección de herramientas y utensilios \\
utilizados? \\
\hline
\end{tabular}

Tabla 1. Entrevista realizada al coordinador del Hato Lechero UFPSO. Fuente: Elaboración propia.

Con la información anteriormente recolectada se procedió a diligenciar una lista de chequeo propuesta para proyectos de ganadería bovina, por la Federación Nacional de Ganaderos (FEDEGAN) en convenio con el Servicio Nacional de
Aprendizaje (SENA). A partir del diligenciamiento de la lista de chequeo mencionada anteriormente, se procedió a realizar una ubicación por puntos georreferenciados, de los focos de contaminación y de consumo de recursos en todo el proyecto, a través de su representación gráfica en un Ecomapa. Adicionalmente se procedió a realizar una matriz de identificación y valoración de impactos ambientales, con el fin de poder evidenciar las posibles afectaciones de mayor significancia y negativas para el medio ambiente, derivadas de los aspectos ambientales más relevantes del proyecto. Con los impactos ambientales generados, se procedió entonces a generar el flujo de procesos del proyecto.

La información recolectada y sistematizada de la forma anteriormente descrita, se llevó a un análisis, para poder determinar las oportunidades, amenazas, fortalezas y debilidades del proyecto y a partir de esto generar unas posibles propuestas de soluciones que permitan superar las debilidades aprovechando las oportunidades y fortalezas y reduciendo las amenazas, todo bajo el marco de los lineamientos de producción más limpia y gestión ambiental, a partir de la corrección múltiples aspectos en el proyecto, ya que este lleva años funcionando en la Granja Experimental de la Universidad, y la posibilidad de formular medidas preventivas para los potenciales aspectos ambientales del mismo, que podrían provocar impactos negativos en el medio.

\section{RESULTADOS}

La entrevista realizada al coordinador del Hato Lechero de la Universidad Francisco de Paula Santander Ocaña, permitió resolver los principales cuestionamientos planteados 
en la misma, los cuales se recopilaron a manera de brindar un panorama ambiental de este importante proyecto.

Este panorama se evidencia en lo siguiente: El Proyecto Hato Lechero se encuentra localizado en las coordenadas $8^{\circ} 14^{\prime} 19.94 " \mathrm{~N}$ y $73^{\circ} 19^{\prime} 17.68^{\prime \prime O}$, en la Granja Experimental de la Sede Algodonal de la Universidad Francisco de Paula Santander Ocaña. Para la producción de leche se cuenta con una infraestructura de aproximadamente $702 \mathrm{~m}^{2}$ de área y un área de 7,59 hectáreas aproximadas, en los cuales se encuentran los bancos de proteínas utilizados para la producción del alimento del ganado y los potreros de pastoreo de los animales, que son 40 exactamente. El hato lechero cuenta con 67 animales, de los cuales 53 son los que producen la leche, estos animales se mantienen en los establos, diferenciados, ya que los utilizados para producción de leche se mantienen en una zona consecutiva a la zona de ordeño, mientras los animales restantes corresponden a lactancia y se mantienen en unos establos ubicados en la zona sur de la infraestructura. Cabe resaltar que el proyecto no cuenta con medidores de consumo de agua ni de energía eléctrica por lo que el cálculo de estos dos factores es indeterminado. Además se generan residuos líquidos los cuales son vertidos a través de un tubo, en la parte sur oeste del proyecto, a pocos metros del mismo, cayendo directamente al suelo e infiltrándose en el mismo. En cuanto a los residuos orgánicos correspondientes a excretas de los animales, gran parte son llevados a los potreros donde se producen especies forrajeras para el abonado de los mismos, acá se apilan y se dejan secar a la intemperie, mientras los restantes residuos de este tipo, son mezclados con cascarilla de arroz y almacenados en costales, los cuales se agrupan y se dejan apilados aledaños a la infraestructura del hato, muy próximos al punto de vertimiento. Actualmente se está empezando a llenar el biodigestor adquirido e instalado para el proyecto, que permitirá el tratamiento de los residuos sólidos orgánicos para posteriormente ser usados como abono en los potreros del mismo, mientras se está adquiriendo un tanque estercolero de plástico para el tratamiento de residuos líquidos. Dentro del funcionamiento del hato, se realiza un ordeño mecánico todos los días en 2 sesiones, de 5:00 a.m. a 6:30 a.m. y de 4:00 p.m. a 5:30 p.m., se mantienen 3 bombillas ahorradoras encendidas desde las 6:00 a.m. hasta las 6:00 p.m. y 6 bombillos adicionales de 5:00 a.m. - 6:00 a.m., se mantiene un motor de 3 caballos de fuerza encendido durante 3 horas al día, a su vez se mantiene un calentador de agua encendido todo el día, y una unidad de enfriamiento de leche durante una hora al día con consumo trifilar y 3 caballos de fuerza. En el mismo proceso de ordeño, para la desinfección de los instrumentos utilizados se utilizan 240 Litros de agua al día, 20 Litros al día para el lavado de los mismos y entre 50 y 80 Litros de agua para el consumo de cada uno de los animales al día. A su vez, los animales del hato que son de la raza Gyrolando en su mayoría, consumen 2 toneladas diarias de forraje, principalmente de los pastos King-Grass, mulato, estrella y guinea. Como se mencionó anteriormente el proyecto Bovino Hato Lechero cuenta con 40 potreros para el pastoreo de los animales, en donde cada potrero se pastoreo cada 40 días. Todos los días se ordeñan 21 vacas por hora, ya que se cuenta con 3 jaulas de ordeño, donde se someten 3 animales a la vez para el proceso, que demora aproximadamente 8 minutos y medio. El litro de leche es vendido a $\$ 1000$, según el coordinador del proyecto, de buena calidad, debido a los análisis de plataforma realizados. Los potreros de todo el proyecto se encuentran divididos por cercas eléctricas, no se han implementado hasta el 
momento cercas vivas y mucho menos sistemas silvopastoriles, pero, a estos potreros se les está realizando verificaciones constantes para visualizar presencia de malezas, que puedan alterar la calidad de los pastos presentes en los mismos, y es por ellos que se realizan aplicaciones de insecticidas y herbicidas una vez al año, en donde la dosificación de herbicidas es de 80 $\mathrm{cm}$ en 20 litros de agua, mientras la de insecticidas depende de las recomendaciones del producto utilizado. La parte sanitaria del proyecto se maneja adaptada a los planes formulados por el ICA, en donde básicamente se controlan enfermedades como la aftosis para la cual se vacunan los animales 2 veces al año, mientras la brúcela se previene vacunando al animal una vez en su vida, entre los 3 a 9 meses después de haber nacido.

El Proceso anterior permitió el diligenciamiento de una lista de Chequeo (ver Tabla 2), propuesta por FEDEGAN y SENA (2011), y adaptada de acuerdo a los aspectos ambientales determinados en el hato lechero.

\begin{tabular}{|c|c|c|}
\hline \multirow[t]{2}{*}{ Factor a tener en cuenta } & \multicolumn{2}{|c|}{ Cumplimiento } \\
\hline & SI & NO \\
\hline Tiene concesión de agua & $\mathrm{X}$ & \\
\hline Tiene análisis de agua & & $\mathrm{X}$ \\
\hline $\begin{array}{l}\text { Calcula la cantidad de agua ideal que } \\
\text { requiere para cada proceso }\end{array}$ & $\mathrm{X}$ & \\
\hline $\begin{array}{l}\text { Revisa y controla que no haya fugas y } \\
\text { desperdicio de agua }\end{array}$ & & $\mathrm{X}$ \\
\hline $\begin{array}{l}\text { Compensa rutinas de aseo entre secas } \\
\text { y con agua }\end{array}$ & & $X$ \\
\hline $\begin{array}{l}\text { Evita que el agua se evapore de } \\
\text { fuentes de agua superficiales }\end{array}$ & & $X$ \\
\hline Evita que el agua se evapore del suelo & & $\mathrm{X}$ \\
\hline $\begin{array}{l}\text { Cuida que los residuos líquidos y } \\
\text { sólidos de las rutinas no contaminen } \\
\text { el agua }\end{array}$ & & $\mathrm{X}$ \\
\hline $\begin{array}{l}\text { Cambia rutinas de tal forma que el } \\
\text { manejo permita disminuir el riesgo de } \\
\text { contaminación del agua }\end{array}$ & & $X$ \\
\hline $\begin{array}{l}\text { Aplica los insumos químicos y } \\
\text { orgánicos de acuerdo con las } \\
\text { recomendaciones evitando que } \\
\text { lleguen a las fuentes de agua }\end{array}$ & $\mathrm{X}$ & \\
\hline
\end{tabular}

Tiene listado de las actividades $\mathrm{y} X$ tiempo oportuno de las rutinas

\begin{tabular}{|c|c|c|}
\hline $\begin{array}{l}\text { Calcula la cantidad de energía que } \\
\text { requiere para cada proceso }\end{array}$ & & $\mathrm{X}$ \\
\hline $\begin{array}{l}\text { Revisa y controla que no haya luces } \\
\text { prendidas }\end{array}$ & $\mathrm{X}$ & \\
\hline $\begin{array}{l}\text { Revisa periódicamente y hace } \\
\text { mantenimiento a su maquinaria } y \\
\text { equipos }\end{array}$ & $\mathrm{X}$ & \\
\hline $\begin{array}{l}\text { Evita que los aparatos trabajen más de } \\
\text { lo normal }\end{array}$ & $\mathrm{X}$ & \\
\hline $\begin{array}{l}\text { Ha explorado alternativas con otras } \\
\text { energías como el sol, el agua y el } \\
\text { viento para sus procesos }\end{array}$ & & $\mathrm{X}$ \\
\hline $\begin{array}{l}\text { Lleva un registro mensual de } \\
\text { combustibles con entradas y salidas }\end{array}$ & & $\mathrm{X}$ \\
\hline $\begin{array}{l}\text { Sabe cuántos jornales necesita para } \\
\text { las diferentes actividades }\end{array}$ & $\mathrm{X}$ & \\
\hline $\begin{array}{l}\text { Ha cuantificado el gasto de energía } \\
\text { para saber cuánto le cuesta en su } \\
\text { producto final }\end{array}$ & & $\mathrm{X}$ \\
\hline $\begin{array}{l}\text { Sus animales están bien tratados y } \\
\text { hacen las rutinas de forma fácil y } \\
\text { segura }\end{array}$ & $\mathrm{X}$ & \\
\hline $\begin{array}{l}\text { Tiene en su finca una dieta } \\
\text { balanceada energéticamente }\end{array}$ & $\mathrm{X}$ & \\
\hline $\begin{array}{l}\text { Hacen sus animales desplazamientos } \\
\text { considerables o inoficiosos }\end{array}$ & & $\mathrm{X}$ \\
\hline $\begin{array}{l}\text { Tiene barreras vivas que evitan } \\
\text { pérdidas o aumento de temperaturas } \\
\text { en los animales (estrés por frío o } \\
\text { calor) }\end{array}$ & & $\mathrm{X}$ \\
\hline $\begin{array}{l}\text { Sustituye materias primas que } \\
\text { generen ahorro de energía }\end{array}$ & & $\mathrm{X}$ \\
\hline $\begin{array}{l}\text { Su finca se encuentra en una finca con } \\
\text { vocación ganadera según el POT }\end{array}$ & $\mathrm{X}$ & \\
\hline Tiene análisis de suelos de su finca & & $\mathrm{X}$ \\
\hline $\begin{array}{l}\text { Calcula la cantidad ideal de animales } \\
\text { que puede tener en su finca de } \\
\text { acuerdo con los diferentes ambientes } \\
\text { de esta }\end{array}$ & & $\mathrm{X}$ \\
\hline $\begin{array}{l}\text { Evita sobrepastorear los potreros en el } \\
\text { invierno }\end{array}$ & $\mathrm{X}$ & \\
\hline $\begin{array}{l}\text { Incorpora materia orgánica a los } \\
\text { suelos }\end{array}$ & $\mathrm{X}$ & \\
\hline Utiliza abonos orgánicos & $\mathrm{X}$ & \\
\hline Utiliza abonos verdes & & $\mathrm{X}$ \\
\hline Hace renovación de praderas & $\mathrm{X}$ & \\
\hline $\begin{array}{l}\text { Hace pruebas de compactación en sus } \\
\text { potreros }\end{array}$ & & $\mathrm{X}$ \\
\hline $\begin{array}{l}\text { Protege las rondas hídricas para evitar } \\
\text { deslizamientos }\end{array}$ & & $\mathrm{X}$ \\
\hline $\begin{array}{l}\text { Protege las áreas susceptibles de } \\
\text { erosión }\end{array}$ & & $\mathrm{X}$ \\
\hline $\begin{array}{l}\text { Utiliza técnicas de ganadería de } \\
\text { conservación para evitar erosión }\end{array}$ & & $\mathrm{X}$ \\
\hline Tiene barreras vivas en sus potreros & & $\mathrm{X}$ \\
\hline Realiza enmiendas de $\mathrm{pH}$ en su finca & & $\mathrm{X}$ \\
\hline $\begin{array}{l}\text { Hace intersiembra de especies en sus } \\
\text { potreros }\end{array}$ & $\mathrm{X}$ & \\
\hline
\end{tabular}




\begin{tabular}{|c|c|c|}
\hline $\begin{array}{l}\text { Hace aforos de sus pastos, cuando } \\
\text { estos están en el momento ideal de } \\
\text { consumo }\end{array}$ & $X$ & \\
\hline $\begin{array}{l}\text { Aplica los insumos químicos y } \\
\text { orgánicos de acuerdo con las } \\
\text { recomendaciones, evitando que } \\
\text { contaminen el suelo }\end{array}$ & $\mathrm{X}$ & \\
\hline $\begin{array}{l}\text { Vigila que sus suelos no se estén } \\
\text { salinizando }\end{array}$ & & $X$ \\
\hline $\begin{array}{l}\text { Sabe cuáles y cuantos son los residuos } \\
\text { del proceso productivo en su finca }\end{array}$ & & $X$ \\
\hline $\begin{array}{l}\text { Hace aplicaciones de fertilizantes y } \\
\text { plaguicidas por observación de } \\
\text { campo, resultados de laboratorio o } \\
\text { sintomatología }\end{array}$ & $X$ & \\
\hline $\begin{array}{l}\text { Evita las quemas de pastos y residuos } \\
\text { en sus cosechas }\end{array}$ & $X$ & \\
\hline $\begin{array}{l}\text { Procura que su producto (leche o } \\
\text { carne) esté libre de residuos de } \\
\text { agroquímicos } \text { o medicamentos } \\
\text { veterinarios }\end{array}$ & $X$ & \\
\hline $\begin{array}{l}\text { Procura que los pastos y forrajes estén } \\
\text { libres de residuos de agroquímicos } \\
\text { que puedan afectar la salud animal }\end{array}$ & $X$ & \\
\hline $\begin{array}{l}\text { Re-usa el agua de los estercoleros } \\
\text { como abono para las praderas }\end{array}$ & & $X$ \\
\hline $\begin{array}{l}\text { Conoce otros usos alternativos para el } \\
\text { manejo o re-uso del estiércol }\end{array}$ & & $X$ \\
\hline $\begin{array}{l}\text { Clasifica y almacena por separado los } \\
\text { residuos que no se pueden re-usar, ni } \\
\text { reciclar }\end{array}$ & $X$ & \\
\hline $\begin{array}{l}\text { Utiliza recipiente guardián para } \\
\text { almacenar residuos cortopunzantes } \\
\text { (agujas, hojas de bisturí y cuchillas } \\
\text { usadas) }\end{array}$ & $X$ & \\
\hline $\begin{array}{l}\text { Hace triple lavado, perfora y entrega } \\
\text { los envases de agroquímicos a las } \\
\text { empresas encargadas }\end{array}$ & $X$ & \\
\hline $\begin{array}{l}\text { Entrega los envases de agroquímicos } \\
\text { y drogas veterinarias a algún } \\
\text { programa de gestión de residuos para } \\
\text { su disposición final }\end{array}$ & $X$ & \\
\hline
\end{tabular}

Tabla 2. Lista de chequeo diligenciada. Fuente: tomada del documento PML en la Ganadería Bovina de la Federación Nacional de Ganaderos \& el SENA, 2011, diligenciada por los autores.

Realizando la respectiva verificación de la situación en la que se encuentra el Hato Lechero, mediante la realización de una entrevista al coordinador del proyecto y el diligenciamiento de una lista de chequeo, se encontraron varios aspectos que a su vez repercutieron en la ocurrencia de impactos ambientales entre los que destacan por su significancia:
- Degradación del suelo por la generación de vertimientos que son dispuestos en este, provenientes de las actividades de ordeño, lavado y desinfección de herramientas y utensilios y limpieza de los establos

- Degradación de la calidad del suelo, por disposición inadecuada de residuos sólidos producto de las actividades de pastoreo de animales y limpieza de los establos.

- Incremento de las concentraciones de gases de efecto invernadero producto de la actividad de pastoreo de los animales

Además de los impactos anteriormente descritos, se determinaron otros impactos los cuales generan poca significancia debido a las condiciones que permiten su ocurrencia. Así, impactos como incremento en la oferta de empleo, afectación a la salud humana por exposición a agroquímicos y disminución de la oferta de agua de la fuente donde se extrae para abastecer el hato, no se consideran relevantes, debido a que no ocurren en una frecuencia mensual como es el caso de la exposición a agroquímicos, cuya ocurrencia es anual, cuando se realizan los controles de malezas, mientras en el caso de la disminución de la oferta de agua de la fuente de donde se extrae, se considera mínimo, debido a que esta agua solo es utilizada para consumo de los animales y esporádicamente para el riego de los bancos de proteínas, y en cuanto al incremento de empleo, que es un impacto positivo, se consideró no significativo, debido a que el proyecto por lo general cuenta con apoyo de los estudiantes de zootecnia, por lo que solo se requiere un profesional que lo coordine, como sucede actualmente, y uno o dos trabajadores para apoyar esporádicamente las actividades de ordeño, limpieza y demás. 
Al identificar los impactos ambientales, se procedió entonces a ubicar en un Ecomapa (Figura 1), los puntos críticos de consumo y contaminación, con el fin de visualizar la ubicación de posibles acciones a futuro que podrían implementarse en el marco de los lineamientos de la Producción Más Limpia.

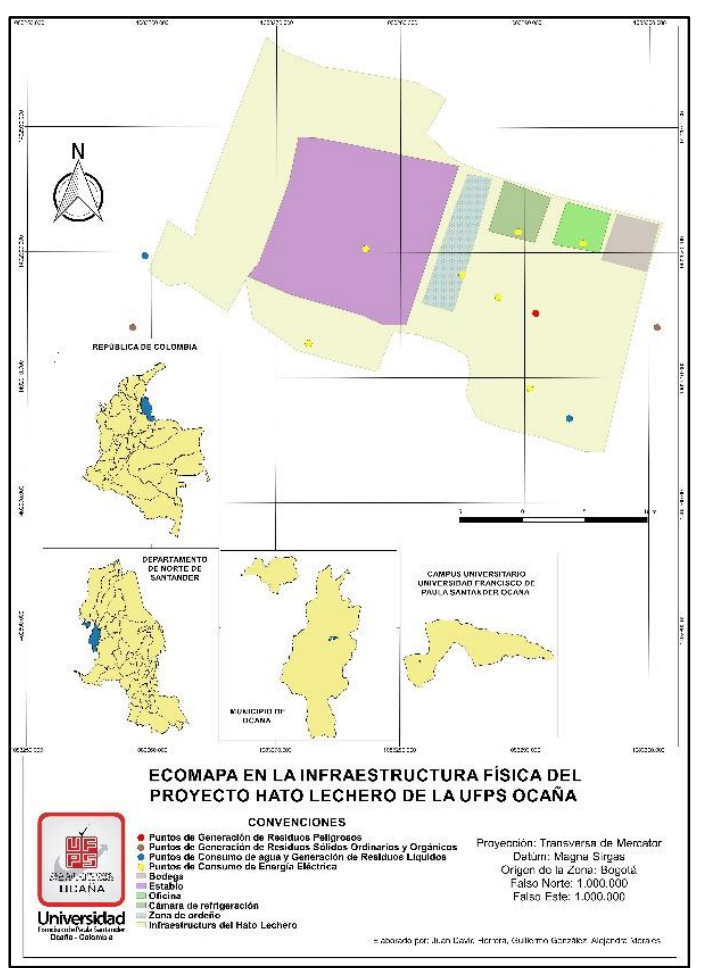

Figura 1. Ecomapa del Proyecto Hato Lechero de la UFPSO. Fuente: Elaboración propia.

Lo anteriormente descrito, permitió la realización del flujo de procesos (Tabla 3).

\begin{tabular}{|c|c|c|}
\hline Entradas & $\begin{array}{l}\text { Operaciones } \\
\text { unitarias }\end{array}$ & Salidas \\
\hline $\begin{array}{l}\text { Animales a } \\
\text { ordeñar } \\
\text { Energía eléctrica }\end{array}$ & Ordeño & $\begin{array}{l}\text { Litros de leche } \\
\text { producidas, } \\
\text { vertimientos, } \\
\text { residuos } \\
\text { peligrosos }\end{array}$ \\
\hline $\begin{array}{l}\text { Agua, } \\
\text { detergente, } \\
\text { desinfectantes }\end{array}$ & $\begin{array}{lr}\text { Lavado } & \text { de } \\
\text { equipos } & y \\
\text { herramientas } & \end{array}$ & $\begin{array}{l}\text { Vertimientos } \\
\text { con detergentes } \\
\text { y desinfectante }\end{array}$ \\
\hline
\end{tabular}

\begin{tabular}{|c|c|c|c|}
\hline Agua & $\begin{array}{l}\text { Limpieza } \\
\text { establos }\end{array}$ & de & $\begin{array}{l}\text { Vertimientos } \\
\text { con residuos } \\
\text { orgánicos, } \\
\text { excretas, } \\
\text { emisiones } \\
\text { atmosféricas }\end{array}$ \\
\hline $\begin{array}{l}\text { Agua, pasto, } \\
\text { suplementos } \\
\text { alimenticios, } \\
\text { suelo }\end{array}$ & $\begin{array}{l}\text { Pastoreo } \\
\text { animales }\end{array}$ & $\mathrm{de}$ & $\begin{array}{l}\text { Excretas, } \\
\text { residuos } \\
\text { líquidos } \\
\text { (orines), suelo } \\
\text { compactado, } \\
\text { emisiones } \\
\text { atmosféricas de } \\
\text { gases, residuos } \\
\text { sólidos } \\
\text { ordinarios }\end{array}$ \\
\hline $\begin{array}{l}\text { Consumo de } \\
\text { agua }\end{array}$ & $\begin{array}{l}\text { Riego } \\
\text { aspersión } \\
\text { potreros }\end{array}$ & $\begin{array}{l}\text { por } \\
\text { de }\end{array}$ & $\begin{array}{ll}\text { Pasturas con } \\
\text { mayor } \\
\text { crecimiento }\end{array}$ \\
\hline $\begin{array}{l}\text { Agroquímicos } \\
\text { (insecticidas y } \\
\text { herbicidas), } \\
\text { Consumo de } \\
\text { agua }\end{array}$ & $\begin{array}{l}\text { Control } \\
\text { malezas } \\
\text { potreros }\end{array}$ & $\begin{array}{l}\text { de } \\
\text { en }\end{array}$ & $\begin{array}{l}\text { Residuos } \\
\text { líquidos con } \\
\text { trazas de } \\
\text { agroquímicos, } \\
\text { emisiones } \\
\text { atmosféricas de } \\
\text { material } \\
\text { particulado y } \\
\text { gases de estos } \\
\text { agroquímicos, } \\
\text { residuos } \\
\text { peligrosos }\end{array}$ \\
\hline $\begin{array}{l}\text { Fármacos, } \\
\text { medicamentos, } \\
\text { vacunas, } \\
\text { elementos } \\
\text { cortopunzantes }\end{array}$ & $\begin{array}{l}\text { Controles } \\
\text { preventivos } \\
\text { correctivos } \\
\text { enfermedades e } \\
\text { animales }\end{array}$ & $\begin{array}{l}\mathrm{y} \\
\mathrm{de} \\
\mathrm{en}\end{array}$ & $\begin{array}{l}\text { Residuos } \\
\text { peligrosos }\end{array}$ \\
\hline
\end{tabular}

Tabla 3. Flujo de Procesos Proyecto Hato Lechero. Fuente: Elaboración propia

\section{DISCUSIÓN}

Al realizar el diagnóstico del proyecto Hato Lechero de la Granja Experimental Sede Algodonal de la UFPSO, se pudo determinar que presenta fortalezas, amenazas, debilidades y oportunidades, que se sintetizan en la matriz DOFA (Tabla 4).

\begin{tabular}{|c|c|}
\hline Debilidades & Oportunidades \\
\hline El proyecto no trata sus & Sistemas silvospastoriles \\
\hline residuos líquidos & Sistematización de los \\
\hline No existe una disposición & procesos \\
\hline $\begin{array}{l}\text { adecuada de los residuos } \\
\text { sólidos }\end{array}$ & $\begin{array}{l}\text { Certificación del ICA en } \\
\text { buenas prácticas ganaderas }\end{array}$ \\
\hline $\begin{array}{l}\text { Se generan emisiones } \\
\text { atmosféricas producto de } \\
\text { las excretas }\end{array}$ & $\begin{array}{l}\text { Buena imagen ante los } \\
\text { productores de la región }\end{array}$ \\
\hline $\begin{array}{l}\text { No se ha planificado } \\
\text { capacidad de carga para los } \\
\text { potreros }\end{array}$ & \\
\hline $\begin{array}{l}\text { No se cuenta con sistemas } \\
\text { silvopastoriles }\end{array}$ & \\
\hline $\begin{array}{l}\text { No se poseen medidores de } \\
\text { agua ni de electricidad }\end{array}$ & \\
\hline
\end{tabular}




\begin{tabular}{|c|c|}
\hline Fortalezas & Amenazas \\
\hline $\begin{array}{l}\text { Certificación del ICA en } \\
\text { proyecto libre } \\
\text { tuberculosis } \\
\text { Infraestructura } \\
\text { adecuada } \\
\text { Rotación de potreros } \\
\text { Área de cuarentena } \\
\text { Producción de leche } \\
\text { Tecnología utilizada para el } \\
\text { ordeño } \\
\text { Razas manejadas dentro del } \\
\text { proyecto } \\
\text { Apoyo de los programas } \\
\text { académicos de Zootecnia e } \\
\text { Ingeniería Ambiental } \\
\text { Apoyo de los Laboratorios } \\
\text { de diagnóstico y } \\
\text { reproducción, nutrición } \\
\text { animal y lácteos y cárnicos }\end{array}$ & $\begin{array}{l}\text { Presencia de otras fincas } \\
\text { ganaderas aledañas, con } \\
\text { desconocimiento de su } \\
\text { estado sanitario } \\
\text { Degradación de la calidad } \\
\text { de las aguas del río } \\
\text { algodonal, aguas arriba del } \\
\text { punto de captación de la } \\
\text { UFPSO } \\
\text { Amenazas por inundación, } \\
\text { debido a la no protección } \\
\text { de rondas hídricas } \\
\text { Amenaza de plagas que } \\
\text { afecten los bancos de } \\
\text { proteínas }\end{array}$ \\
\hline
\end{tabular}

Tabla 4. Matriz DOFA Proyecto Hato Lechero. Fuente: Elaboración propia.

Como se puede evidenciar, el proyecto presenta una constante verificación de sus procesos sanitarios, con el fin de generar productos de calidad los cuales ofertan a la población en general. Sin embargo este proyecto, que a su vez, se encuentra inmerso dentro del Proyecto Bovino de la Universidad, es el único de especies mayores, de los 5 proyectos pecuarios que maneja la Granja Experimental, y adicionalmente es el proyecto que por obvias razones, genera los mayores consumos no solo de materias primas, sino también de agua, energía eléctrica e insumos de tipo veterinario, que ocasionan la mayor cantidad de residuos líquidos, sólidos, gaseosos (GEI) y peligrosos, de los cuales hasta el momento solo se manejan y disponen adecuadamente, los de tipo peligrosos, ya que están vinculados con una de las principales líneas de acción del Sistema de Gestión Ambiental de la Universidad, la cual es la disposición adecuada de este tipo de residuos, muy frecuentes en el campus universitario, debido a la presencia de laboratorios que los generan y de los proyectos de la granja.

Por otra parte, para realizar un diagnóstico más profundo de la situación ambiental que presenta el proyecto, es necesario la instalación de aparatos que controlen los consumos del mismo. Es así como se hace necesario la instalación de medidores de consumo de agua, puesto que una parte del proyecto se abastece del agua captada directamente del Río Algodonal, en lo que tiene que ver con consumo de agua de los animales y riego de los potreros, a diferencia de las actividades de ordeño y limpieza y desinfección de herramientas y utensilios, los cuales consumen agua potable, suministrada por la Empresa ESPO S.A. la cual opera el acueducto del municipio de Ocaña. Adicional mente se deben instalar medidores de electricidad, ya que no existen, y considerando la cantidad de equipos que se manejan dentro de la infraestructura del Hato, más la energía que consumen las cercas eléctricas en los potreros, se hace necesario conocer estos datos de consumo para poder estipular medidas de ahorro de electricidad que sean eficaces.

Considerando las condiciones del proyecto, el cual hace parte de una Universidad como la UFPSO, que cuenta con carreras interdisciplinares, se debe aprovechar el potencial humano y tecnológico de las mismas, con el fin de generar un proceso de sistematización del Hato, en donde se desarrolle un software de registro de materias primas que por supuesto entran al proyecto, y de las materias primas que hacen falta con el fin de generar los reportes de compra, complementando dicho software con el registro de los pesos de los animales, para lo cual también es necesario una báscula, y el registro de la cantidad de residuos sólidos y peligrosos generados en el proyecto, como también los volúmenes de residuos líquidos. En el caso de los residuos sólidos de tipo orgánico y líquidos, se debe tener en cuenta y agilizar el proceso de funcionamiento del biodigestor instalado $\mathrm{y}$ de compra del tanque estercolero, a partir de 
los cuales se realice un procedo de tratamiento adecuado de estos residuos, para posteriormente obtener abonos y gases generadores de energía eléctrica, que permitan ser utilizados de forma sostenible en el abonado de potreros, y para abastecer de energía eléctrica el proyecto, puesto que específicamente los gases generados en el biodigestor, pueden tener un potencial para generar energía eléctrica, la cual puede ser almacenada en una planta eléctrica y utilizarse para abastecer los distintos equipos eléctricos que integran la infraestructura del hato y que se utilizan regularmente para el ordeño y procesamiento de la leche. El software planteado debe registrar todo lo anterior, pero también debe registrar las producciones de leche, y las posibles compras directas del producto, registrando además los controles de enfermedades de los animales y los controles de malezas a potreros.

Es necesario que como otra medida de control, se realicen verificaciones de las condiciones de los suelos de los potreros, con el fin de tomar acciones preventivas para su mantenimiento, siendo necesario además, su fertilización con abonos orgánicos y abonos verdes, y el establecimiento de los tiempos requeridos para la rotación de potreros y la capacidad de carga de estos suelos, acciones que se pueden complementar con el establecimiento de cercas vivas y sistemas silvopastoriles en los potreros donde pastorean los animales del Hato, con el fin de lograr una conservación adecuada de los suelos, y de las pasturas, y brindar además un bienestar a los animales, que se evidencie en su producción y obviamente en su estado de salud, fomentándose así prácticas de bioética animal dentro de la granja experimental, que sirvan de ejemplo para los productores de la región y para la academia de la institución.

\section{CONCLUSIONES.}

Es necesaria la implementación de prácticas de producción más limpia en el proyecto Hato Lechero de la UFPSO. Sin embargo como primera medida se debe profundizar en el diagnóstico del mismo, puesto que no existen mecanismos que permitan establecer unos balances de materia y energía completos, mediante los cuales se definan las medidas de mayor pertinencia para el proyecto. El Proyecto tiene una trayectoria larga en la Granja Experimental de la Universidad, pero para seguir manteniendo esa trayectoria y ser sustentable, debe cambiar varios de sus procedimientos, que le permitirán adentrarse aún más en el tema de la calidad, el cual actualmente busca consolidar a través de una aspiración a certificado de buenas prácticas ganaderas del ICA, y esto se logra no solo desde el punto de vista de la zootecnia, sino también vinculando el punto de vista ambiental, que en estos momentos no se han tenido en cuenta de la manera adecuada. La mayor de las fortalezas del proyecto es el potencial humano, financiero, tecnológico e intelectual con el que cuenta la Universidad, y que está a su disposición, solo se necesita mayor articulación para poder implementar procesos tendientes a la prevención de la contaminación a partir de un uso eficiente de las materias primas que entran al Hato Lechero, así como un manejo adecuado de sus residuos, que le permitan ser autosostenible y no representar una carga más para la Universidad, generando a su vez una imagen de calidad en el interior de la Institución y ante los productores de la región, y consolidándose como ejemplo de implementación de Producción Más Limpia para las demás fincas que se dedican a la producción de leche en Ocaña y sus alrededores. La Universidad ya se encuentra por un buen camino con la implementación 
de su Sistema de Gestión Ambiental, solo es necesario implementar la Producción Más Limpia como la principal herramienta de ese Sistema, y especialmente en los proyectos de la Granja Experimental que consumen tanta materia prima, y generan a su vez contaminación al medio ambiente.

\section{FINANCIACIÓN}

A la Universidad Francisco de Paula Santander Ocaña, a la Granja Experimental $\mathrm{y}$ en especial al profesional que apoyan el Proyecto Bovino - Hato Lechero de la UFPSO, por toda su colaboración en el proceso de diagnóstico situacional del proyecto.

\section{BIBLIOGRAFÍA}

Arboleda-González, J. A. (2008). Manual para la Evaluación de Impacto Ambiental de Proyectos, Obras o Actividades. Medellín, Colombia. Recuperado el 10 de Junio de 2015, de

http://evaluaciondelimpactoambienta 1.bligoo.com.co/media/users/20/1033 390/files/255491/1_Manual_EIA.pdf

Bichachi, D. S. (2003). El uso de las Listas de Chequeo ( CheskList) como herramienta para controlar la calidad de la ley. Humahuaca, Argentina. Recuperado el 16 de Junio de 2015, de

http://claudiabernazza.com.ar/htm/pd f/check_list.pdf

Camacho-Fidalgo, A. (2011). Aplicando Producción Más Limpia en el Sector Lechero. Recuperado el 11 de Junio de 2015, de http://www.cegesti.org/exitoempresa rial/publicaciones/publicacion_144_ 050411_es.pdf
Congreso de la República . (24 de Enero de 1979). Alcaldía de Bogotá . Obtenido de

http://www.alcaldiabogota.gov.co/sis jur/normas/Norma1.jsp?i=1177

Congreso de la República . (27 de Diciembre de 1989). Alcaldía de Bogotá . Obtenido de http://www.alcaldiabogota.gov.co/sis jur/normas/Norma1.jsp?i=8242

Congreso de la República . (6 de Junio de 1997). Alcaldía de Bogotá. Obtenido de

http://www.alcaldiabogota.gov.co/sis jur/normas/Norma1.jsp?i=342

Congreso de la República . (16 de Enero de 1998). Universidad del Externado, Medio Ambiente . Obtenido de http://medioambiente.uexternado.edu .co/m3d10AmB/wpcontent/uploads/2015/04/Ley-430de-1998.pdf

Congreso de la República . (3 de Julio de 2003). Instituto Colombiano Agropecuario . Obtenido de http://www.ica.gov.co/getattachment /c7999637-49d8-4f2d-99dfe5ebb065f896/2003L822.aspx

Congreso de la República . (21 de Octubre de 2004). Instituto Colombiano Agropecuario . Obtenido de http://www.ica.gov.co/getattachment /8b30fb3e-26f1-48f3-a738$54 \mathrm{fb} 7 \mathrm{~d} 06 \mathrm{c} 8 \mathrm{a} 9 / 2004 \mathrm{~L} 914-$ $\% 281 \% 29$.aspx

Congreso de la República. (23 de Diciembre de 1993). Alcaldía de Bogotá. Obtenido de http://www.alcaldiabogota.gov.co/sis jur/normas/Norma1.jsp?i=297 
Congreso de la República. (2 de Agosto de 1997). Instituto Colombiano Agropecuario. Obtenido de http://www.ica.gov.co/getattachment /e73275d2-b626-458b-98f1fea3d80ef95f/395.aspx

Federación Colombia de Ganaderos. (2011). Producción Más Limpia - PML en la Ganadería Bovina. Bogotá D. C.: FEDEGAN. Recuperado el 4 de Mayo de 2015, de http://www.slideshare.net/Fedegan/p roduccion-mas-limpia-27700647

Garzón-Benavides, J. M., \& López-Morán, J. M. (2008). Análisis de una alternativa de producción más limpia que permita aprovechar los residuos grasos que generan los procesos de pasteurización y enfriamiento de la leche en la empresa Friesland Lacteos Purace de San Juan de Pasto. Pereira, Risaralda, Colombia. Recuperado el 08 de Junio de 2015, de

http://repositorio.utp.edu.co/dspace/b itstream/11059/1539/1/62844G245.p df

Guédez-Mozur, C., Armas-Hernández, D., Reyes-Gil, R., \& Galván-Rico, L. (Septiembre de 2003). Los sistemas de gestión ambiental en la industria petrolera internacional. Interciencia , 28(9), 528-533. Recuperado el 13 de Junio de 2015, de http://www.redalyc.org/articulo.oa?i $\mathrm{d}=33908406$

Isaza-Vargas, M. F. (2012). Compromiso Ambiental y Sustentabilidad de la Industria de Alimentos Lácteos en Colombia. Bogotá, D.C., Colombia. Recuperado el 12 de Junio de 2015, de http://repository.unimilitar.edu.co/bit
stream/10654/10498/1/IsazaVargas

MariaFernanda2013.pdf

Loaiza-Bedoya, Y. A., \& Osorio-Montoya, A. L. (2009). Gestión del agua en el sector de la ganadería bovina en la cuenca río la Vieja departamentos de Quindío y Risaralda. Pereira, Risaralda, Colombia. Recuperado el 05 de Junio de 2015, de https://www.google.com.co/url?sa=t $\& \mathrm{rct}=\mathrm{j} \& \mathrm{q}=\&$ esrc $=\mathrm{s} \&$ source $=$ web $\& \mathrm{c}$ $\mathrm{d}=3 \&$ cad $=$ rja \&uact $=8 \&$ ved $=0 \mathrm{CCYQ}$ FjAC\&url=http $\% 3 \mathrm{~A} \% 2 \mathrm{~F} \% 2$ Freposit orio.utp.edu.co $\% 2 \mathrm{Fdspace} \% 2 \mathrm{Fbitstre}$ am\%2F11059\%2F1195\%2F1\%2F62 81586132L795.pdf\&ei=CAODVaj8 K7X7sATYuKvoDQ\&usg=AFQjCN HQ3rkfHCx6mi6quYetq5nQ

Medina-Orna, S. X., \& Zea-Vargas, D. F. (2005). Implementación de la guía metodológica de producción más limpia en el sector hotelero, estudio de caso Hacienda El Royal. Bogotá, D. C., Colombia. Recuperado el 14 de Junio de 2015, de http://repository.lasalle.edu.co/bitstre am/handle/10185/14727/00798116.p df? sequence $=1$

Organización de las Naciones Unidas para la Alimentación y la Agricultura (FAO). (2012). Guía de buenas prácticas en explotaciones lecheras. Roma, Italia. Recuperado el 10 de Junio de 2015, de http://www.fao.org/docrep/015/ba00 27s/ba0027s00.pdf

Penella, F. (Septiembre de 2012). Producción Más Limpia. Argentina. Recuperado el 09 de Junio de 2015, de

http://www.ambiente.gov.ar/archivos /web/Ppnud08/file/Clase\%207_\%20I 
ng_\%20Fabio\%20Pennella_Producci

$\% \mathrm{C} 3 \% \mathrm{~B} 3 \mathrm{n} \% 20$ mas\%20Limpia.pdf

Varón-Jiménez, L. (2013). La producción más limpia como estrategia de gestión ambiental. Producción + Limpia, 8(1), 1. Recuperado el 12 de Junio de 2015, de http://www.scielo.org.co/scielo.php? pid=S1909-

04552013000100001\&script=sci_artt ext 